\title{
Extended Model Formulation of the Proportional Lot-Sizing and Scheduling Problem with Lost Demand Costs
}

\author{
Waldemar Kaczmarczyk ${ }^{\star}$
}

\begin{abstract}
We consider mixed-integer linear programming (MIP) models of production planning problems known as the small bucket lot-sizing and scheduling problems. We present an application of a class of valid inequalities to the case with lost demand (stock-out) costs. Presented results of numerical experiments made for the the Proportional Lot-sizing and Scheduling Problem (PLSP) confirm benefits of such extended model formulation.
\end{abstract}

Keywords: production, lot-sizing and scheduling, mixed integer linear programming

Mathematics Subject Classification: 90B30, 90C11

Revised: 27 August 2011

\section{INTRODUCTION}

In this paper we consider mixed-integer linear programming (MIP) models of lot-sizing and scheduling problems, which assume that several products with deterministic, dynamic demand, have to be produced alternately on the same single machine with limited capacity. In each period, the total workload assigned to the machine cannot exceed its capacity. When the machine is changed over from production of one product to another a set-up operation must be executed. The demand does not have to be satisfied on the whole, but unsatisfied, lost demand rises costs proportional to its size. Unsatisfied demands (sales) are called lost. The objective is to minimize the sum of the lost demand costs, set-up and inventory holding costs in all periods.

Most production lost-sizing problems assume complete fulfilment of all demands. This is however not always justified. In stochastic lot-sizing problems lost demand (stock-out) costs arise when demand occurs while products are out of stock. In deterministic production planning problems, lost demand costs may occur for several reasons. First of all, this may be unavoidable if there is not enough production or storage capacity. Secondly, in some circumstances shortage costs may be smaller then the sum of production, setup and inventory holding costs.

* AGH University of Science and Technology, Faculty of Management, Department of Operations Research and Information Technology, Krakow, Poland. E-mail: waldek@agh.edu.pl 
The problem under consideration is motivated by a real-world case in the automobile industry. In industrial supply chains, in vendor managed inventory (VMI) system, when client declares some demand too late, supplier is not obligated to satisfy this additional demand. Such late orders may have however higher price what may be for the supplier a strong incentive to check, how much of this additional demand is he able to fulfill. Sometimes supplier wants to fulfill as much as possible of such late orders just for the sake of good cooperation and sets prohibitive values of lost demand unit costs.

Companies may avoid shortages by backordering or outsourcing. Some companies adjust prices to match the demand and supply, i.e. use techniques of the revenue management. All these methods to prevent lost demand are out of scope of this paper.

The Proportional Lot-sizing and Scheduling Problem (PLSP) was proposed by Haase (1994), see also Drexl and Haase (1995). It is a small bucket model, i.e. it allows only one set-up operation within a single period. The PLSP is the most flexible small bucket model, because it allows the processing of two products in a single period, one before and one after the set-up operation. It has been extended for several special cases, among others for the case with long (multi period) setup times (Suerie, 2005; Kaczmarczyk, 2009a) and for the case with identical parallel machines (Kaczmarczyk, 2011). General surveys on lot-sizing and scheduling models can be found in Drexl and Kimms (1997) or Jans and Degraeve (2008).

Objective of an extended model formulation is to make a model more tight, i.e. to make it easier to solve with standard MIP methods. Valid inequalities are constraints added the model although they are not necessary to make it more realistic. Their objective is to tighten linear relaxation of the model, what improves efficiency of the branch and bound algorithm. Valid inequalities may be added a priori to the model or dynamically generated by the branch and cut algorithm. A review of various extended model formulations for many lot-sizing problems can be found in Belvaux and Wolsey (2001), Wolsey (2002) or Pochet and Wolsey (2006).

Most valid inequalities applied to production lot-sizing problems were originally developed for the classic Uncapacitated Lot-Sizng Problem (ULSP) described by Wagner and Whitin (1958). For example Barany et al. (1984) proposed a class of valid inequalities and proved that the ULSP extended by this constraints is fully tight, i.e. can be solved with pure (non-integer) linear programming algorithm. Such inequalities are useful also for models with several products, however they do not make them fully tight. Models of the lot-sizing and scheduling problems may be tightened among others by adding a class of valid inequalities which set lower bounds on inventory levels dependent on the length of time intervals in which production will not be executed (Belvaux and Wolsey, 2001). This paper presents an application of these inequalities in the case with lost demand costs.

There are a few papers on production lot-sizing and scheduling dealing with lost demand. Sandbothe and Thompson (1990) proposed a forward dynamic programming algorithm for a single product model with limited capacity. Aksen et al. (2003) proposed a dynamic programming algorithm for the ULSP. Aksen (2007) adopted this model to the case when unsatisfied demand in one period causes the demand in the following period to decrease due to the loss of customer goodwill. 
Liu et al. (2007) consider a single product dynamic lot sizing problem with limited storage capacity and lost sales. Berk et al. (2008) propose a dynamic programming formulation for a single item lot-sizing problem with lost sales for the case with warm and cold setups. The machine can be kept warm, i.e. ready to work, for the next period only if more than a minimal amount of product is produced. Cold machine, i.e. after longer pause, has to be set-up. Lu and Qi (2011) consider a joint production of multiple products with lost sales and without capacity limit.

Two papers consider extended model formulations for production lot-sizing problems with lost demand cost. Loparic et al. (2001) present tight formulations of the ULSP with sales variables with an upper limit. They describe two equivalent model formulations: first, with inventory lover bounds, second, with positive or negative demands that have to be satisfied. Absi and Kedad-Sidhoum (2008) propose valid inequalities for the multi-item capacitated lot-sizing problem with setup times and shortage costs and apply them within a branch and cut algorithm.

The next section presents the PLSP model with lost demand. Section 3 presents applied valid inequalities. Finally, section 4 shows computational results for standard and extended PLSP model.

\section{STANDARD MODEL}

The dynamic lot-sizing problem, also called the Wagner-Whitin problem (Wagner and Whitin, 1958), assumes a discrete planning horizon, i.e. the time is modelled as a finite number of time points. The intervals between the time points are called time periods or time buckets. Demand is given per period and varies over time. In this paper, models are discussed which adopt the dynamic lot-sizing problem for the case with many products and a limited capacity of resources. They are usually called lot-sizing and scheduling problems. One of them is the PLSP. Its parameters and variables in the case with lost demand are presented in Tables 1 and 2 .

Table 1. Parameters.

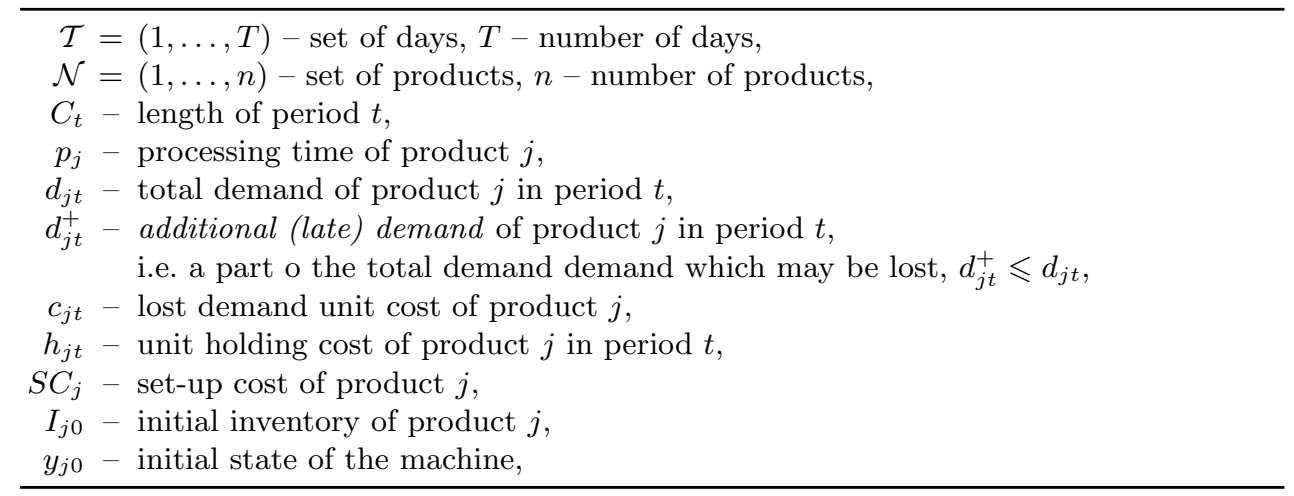

All variables with period index $t \leqslant 0$ are assumed to be equal to 0 . There are only two exceptions. First, the inventory variables $I_{j 0}$ represent non-zero initial inventories. Second, the set-up state variables $y_{j 0}$ define the initial state of the machine. 
Table 2. Variables.

$I_{j t}-$ inventory of product $j$ in period $t$,

$x_{j t}-$ production volume of product $j$ in period $t$,

$v_{j t}-$ demand of product $j$ lost in period $t, v_{j t} \leqslant d_{j t}^{+}$,

$y_{j t}=1$, if at the end of period $t$ machine is set up for product $j, 0$ otherwise,

$z_{j t}=1$, if in period $t$ machine starts up to process product $j, 0$ otherwise,

A mixed-integer programming model of the PLSP with lost demand can be stated as follows:

$$
\begin{array}{cl}
\min \sum_{t \in \mathcal{T}} \sum_{j \in \mathcal{N}}\left(S C_{j} z_{j t}+h_{j t} I_{j t}+c_{j} v_{j t}\right) & \\
I_{j t-1}+x_{j t}=d_{j t}-v_{j t}+I_{j t}, & t \in \mathcal{T}, j \in \mathcal{N} \\
p_{j} x_{j t} \leqslant C_{t}\left(y_{j t-1}+y_{j t}\right), & t \in \mathcal{T}, j \in \mathcal{N} \\
\sum_{j \in \mathcal{N}} p_{j} x_{j t} \leqslant C_{t}, & t \in \mathcal{T} \\
\sum_{j \in \mathcal{N}} y_{j t}=1, & t \in \mathcal{T} \\
y_{j t}-y_{j, t-1} \leqslant z_{j t}, & t \in \mathcal{T}, j \in \mathcal{N} \\
0 \leqslant v_{j t} \leqslant d_{j t}^{+}, & t \in \mathcal{T}, j \in \mathcal{N} \\
I_{j t}, x_{j t} \geqslant 0, & t \in \mathcal{T}, j \in \mathcal{N} \\
z_{j t}, y_{j t} \in\{0,1\}, & t \in \mathcal{T}, j \in \mathcal{N}
\end{array}
$$

The objective function (1.1) represents the total set-up, inventory and lost demand costs. Constraint (1.2) describes the balance of inventory, production and fulfilled demand, i.e. the total demand reduced by the lost demand. Constraint (1.3) allows production to take values higher than zero only if the machine is set up to process a given product in the current or previous period. Inequality (1.4) ensures that the total workload does not exceed capacity of a single period. Constraint (1.5) ensures that in every period, the set-up state variable of only one product takes the value one, i.e. the machine is set up to process exactly one product. Inequality (1.6) ensures that start-up variables are equal to one if the machine is set up to process a given product in the current period but not in the previous period.

\section{VALID INEQUALITIES}

Valid inequalities are constraints which are not essential to define a realistic MIP model of the considered system, but make more tight its linear relaxations (Belvaux and Wolsey, 2001;Wolsey, 2002; Pochet and Wolsey, 2006). In optimal solutions of a tight linear relaxation of a MIP model relaxed (integer) variables are more likely to 
take integer values. This way valid inequalities reduce the number of nodes needed to solve a MIP problem and may speed up execution of the branch and bound algorithm.

In several papers on lot-sizing and scheduling (e.g. Suerie, 2005; Kaczmarczyk; 2009) authors add a priori to the model valid inequalities, recommended by Belvaux and Wolsey (2001), which determine the lower bound on inventory level in period $t-1$ which is necessary to satisfy the demand from period $t$ to period $t+p$. The time interval $[t, t+p]$ may be interpreted as a run-out time of lots finished in period $t-1$ or earlier. Below introduced is an adaptation of these inequalities to the case with lost demand.

If the machine was not set up to process product $j$ in period $t-1$ and it is not started up to process product $j$ in period $t$, then in period $t$ product $j$ can not be produced and the total demand decreased by the lost demand has to be fulfilled from inventory build up at the end of period $t-1$. This condition is expressed by following constraint:

$$
I_{j, t-1} \geqslant d_{j t}\left(1-v_{j t} / d_{j t}-y_{j, t-1}-z_{j, t}\right)
$$

where $v_{j t} / d_{j t}$ is the fraction of demand lost in period $t$.

If the machine will not be started-up to produce product $j$ also in period $t+1$, then also the demand from period $t+1$ will be fulfilled from inventory from period $t-1$, what can be described by following condition:

$$
\begin{aligned}
I_{j, t-1} & \geqslant d_{j t}\left(1-v_{j t} / d_{j t}-y_{j, t-1}-z_{j, t}\right) \\
& +d_{j, t+1}\left(1-v_{j, t+1} / d_{j, t+1}-y_{j, t-1}-z_{j, t}-z_{j, t+1}\right)
\end{aligned}
$$

If the time interval without any start-ups for product $j$ lasts over $p$ periods then the above constraint can be generalized in the following way:

$$
\begin{aligned}
I_{j, t-1} \geqslant \sum_{s=t}^{t+p} d_{j s}\left[1-v_{j s} / d_{j s}-y_{j, t-1}-\sum_{r=t}^{s} z_{j r}\right], \\
t \in \mathcal{T}, p=0, \ldots, \min \left\{P_{\max }-1, T-t\right\}, j \in \mathcal{N}
\end{aligned}
$$

In general run-out time intervals may end in the last period $T$. In inequalities (2) their length is limited by $P_{\max }$, because for every product there are usually a few start-ups within the whole planing horizon and the run-out time intervals do not end in $T$. Therefore it is not practical to add all possible valid inequalities o type (2) a priori to the model (Kaczmarczyk; 2009b). $P_{\max }$ values may be based on experience.

Following valid inequalities (Belvaux and Wolsey, 2001; Wolsey, 2002) are usually added a priori to multiproduct lot-sizing and scheduling models and were used in experiments described in the next section:

$$
\begin{array}{ll}
z_{j t} \leqslant y_{j t}, & t \in \mathcal{T}, j \in \mathcal{N} \\
z_{j t} \leqslant 1-y_{j, t-1}, & t \in \mathcal{T}, j \in \mathcal{N}
\end{array}
$$

Constraint (3a) resets start-up variables in periods without set-up. Inequality (3b) resets start-up variables if in the previous period the machine was already set up. 


\section{RESULTS OF EXPERIMENTS}

To verify the impact of valid inequalities on the PLSP, the following experiments were executed. Five data sets were randomly generated according to the following procedure. All problem instances had 30 periods and 5 products.

At first, all of the demands $d_{j t}$ were drawn from the range $\left[10 \alpha_{j}, 100 \beta_{j}\right]$, where $\alpha_{j} \in\{1, \ldots, 5\}$ and $\beta_{j} \in\{1,2,3\}$. Next each of them was set equal to 0 with probability $0.1 \gamma_{j}$ where $\gamma_{j} \in\{2, \ldots, 8\}$. Also the demand of the first four days for all products was set equal to 0 .

Processing times $p_{j}$ of all products were equal to 1 . The capacity of the machine $C$ was chosen in such a way as to keep the workload of the machine equal to $80 \%$. Unit holding costs $h_{j}$ were randomly chosen from the set $\{1, \ldots, 5\}$. The set-up cost was calculated as $S C_{j}=\lambda_{j} * h_{j} * C$, where $\lambda_{j}$ was randomly chosen from the set $\{1, \ldots, 15\}$.

All instances have been solved for the standard (eq. (1), (3)) and extended (eq. (1), (3), (2)) model formulation of the PLSP with $P_{\max }=7$, for set-up costs equal to $100 \%, 25 \%$ or $400 \%$ of the originally generated costs $S C_{j}$, and for the unit lost demand costs 100 or 20 times higher then the unit holding costs of the same product.

In Table 3 the results for both models are presented. Here, the MIP gap is defined as $\left(f^{*}-L B\right) / L B$, where $f^{*}$ is the best value found for the objective function, and $L B$ is the lower bound. The total cost surplus shows the relative difference between the objective value found with given model and the best value found with both models.

All tests were performed with a 5 minute time limit on an Intel Core i7 processor with a $2.8 \mathrm{MHz}$ clock speed and 8 GB RAM running ILOG OPL Studio 6.3 (CPLEX 12) with standard settings.

Table 3. Average computational results.

\begin{tabular}{|c|c|c|c|c|c|c|c|}
\hline \multirow{3}{*}{\multicolumn{2}{|c|}{$\begin{array}{r}\text { Model } \\
\text { Lost demand } \\
\text { unit cost }\end{array}$}} & \multicolumn{3}{|c|}{ standard } & \multicolumn{3}{|c|}{ extended } \\
\hline & & \multicolumn{3}{|c|}{ Setup cost } & \multicolumn{3}{|c|}{ Setup cost } \\
\hline & & $25 \%$ & $100 \%$ & $400 \%$ & $25 \%$ & $100 \%$ & $400 \%$ \\
\hline \multirow[t]{2}{*}{ MIP gap } & 100 & $70.2 \%$ & $37.1 \%$ & $17.0 \%$ & $20.1 \%$ & $10.6 \%$ & - \\
\hline & 20 & $26.1 \%$ & $15.4 \%$ & - & $3.1 \%$ & $0.5 \%$ & - \\
\hline \multirow[t]{2}{*}{ Time $[\mathrm{s}]$} & 100 & 300 & 300 & 266 & 300 & 252 & 114 \\
\hline & 20 & 300 & 300 & 22 & 290 & 178 & 5 \\
\hline \multirow{2}{*}{$\begin{array}{l}\text { Total cost } \\
\text { surplus }\end{array}$} & 100 & $8.6 \%$ & $4.2 \%$ & $0.4 \%$ & - & $0.7 \%$ & - \\
\hline & 20 & $3.0 \%$ & $0.6 \%$ & - & - & - & - \\
\hline \multirow[t]{2}{*}{ Lost demand } & 100 & $2.5 \%$ & $1.7 \%$ & $3.9 \%$ & $2.2 \%$ & $2.0 \%$ & $3.1 \%$ \\
\hline & 20 & $9.8 \%$ & $10.5 \%$ & $46.0 \%$ & $10.4 \%$ & $10.2 \%$ & $45.8 \%$ \\
\hline
\end{tabular}

Dash is substituted for zero

The results prove that the extended model is superior to the standard model. For low workload and small setup costs the solver is usually not able to find optimal solutions within assumed time limit, therefore the MIP gap is high and computation time close to the time limit. In such cases extended model ensures up to $8.6 \%$ better solutions. 
For high workload and large setup costs the solver more often determines optimal solutions, however much faster for the extended model than for the standard model.

High lost demand unit costs represent the case when company tries to minimize the lost demand in first place. In this case the extended model ensures a little smaller values of lost demand. For low lost demand unit cost it may be profitable to increase lost demand to avoid some setup and inventory holding costs. Therefore the total lost demand takes high values. Low lost demand unit cost and high setup cost lead to large values of the total lost demand that are not realistic, i.e. unacceptable for any company.

\section{REFERENCES}

Absi, N., Kedad-Sidhoum, S., 2008. The multi-item capacitated lot-sizing problem with setup times and shortage costs. European Journal of Operational Research 185, 1351-1374.

Aksen, D., 2007. Loss of customer goodwill in the uncapacitated lot-sizing problem. Computers 85 Operations Research 34, 2805-2823.

Aksen, D., Altınkemer, K., Chand, S., 2003. The single-item lot-sizing problem with immediate lost sales. European Journal of Operational Research 147, 558-566.

Barany, I., van Roy, T. J., 1984. Uncapacitated lot-sizing: The convex hull of solutions. Mathematical Programming Studies 22, 32-43.

Belvaux, G., Wolsey, L.A., 2001. Modelling practical lot-sizing problems as mixed-integer programs. Management Science 47(7), 993-1007.

Berk, E., Toy, A. Ö., Hazır, Ö., 2008. Single item lot-sizing problem for a warm/cold process with immediate lost sales. European Journal of Operational Research 187, 1251-1267.

Drexl, A., Haase, K., 1995. Proportional lotsizing and scheduling. International Journal of Production Economics 40, 73-87.

Drexl, A., Kimms, A., 1997. Lot sizing and scheduling - survey and extensions. European Journal of Operational Research 99(2), 221-235.

Haase, K., 1994. Lotsizing and scheduling for production planning. In Lecture Notes in Economics and Mathematical Systems, number 408, Springer-Verlag.

Jans, R., Degraeve, Z., 2008. Modeling industrial lot sizing problems: a review. International Journal of Production Research 46(6), 1619-1643.

Kaczmarczyk, W., 2009a. Modelling multi-period set-up times in the proportional lot-sizing problem. Decision Making in Manufacturing and Services 3(1-2), 15-35.

Kaczmarczyk, W., 2009b. Practical Tips for Modelling Lot-Sizing and Scheduling Problems. Decision Making in Manufacturing and Services 3, 37-48.

Kaczmarczyk, W., 2011. Proportional lot-sizing and scheduling problem with identical parallel machines. International Journal of Production Research 49(9), 2605-2623.

Liu, X., Chu, F., Chu, C., Wang C., 2007. Lot sizing with bounded inventory and lost sales. International Journal of Production Research 45(24), 5881-5894.

Loparic, M., Pochet Y., Wolsey, L. A., 2001. The uncapacitated lot-sizing problem with sales and safety stocks. Mathematical Programming 89, 487-504.

Lu, L., Qi, X., 2011. Dynamic lot sizing for multiple products with a new joint replenishment model. European Journal of Operational Research 212, 74-80.

Pochet, Y., Wolsey, L.A., 2006. Production planning by mixed integer programming. Series in Operations Research and Financial Engineering, Springer. New York.

Sandbothe, R. A., Thompson, G. L., 1990. A Forward Algorithm For The Capacitated Lot Size Model With Stockouts. Operations Research 38(3), 474-486. 
Suerie, C., 2005. Time Continuity in Discrete Time Models, Springer.

Wagner, H.M., Whitin, T.M., 1958. Dynamic version of the economic lot size model. Management Science 5, 89-96.

Wolsey, L. A., 2002. Solving multi-item lot-sizing problems with an MIP solver using classification and reformulation. Management Science 48(12), 1587-1602. 